\title{
Semantically-based crossover in genetic programming: application to real-valued symbolic regression
}

\author{
Nguyen Quang Uy • Nguyen Xuan Hoai • \\ Michael O’Neill • R. I. McKay • Edgar Galván-López
}

Received: 25 December 2009/Revised: 1 July 2010/Published online: 30 July 2010

(C) Springer Science+Business Media, LLC 2010

\begin{abstract}
We investigate the effects of semantically-based crossover operators in genetic programming, applied to real-valued symbolic regression problems. We propose two new relations derived from the semantic distance between subtrees, known as semantic equivalence and semantic similarity. These relations are used to guide variants of the crossover operator, resulting in two new crossover operatorssemantics aware crossover (SAC) and semantic similarity-based crossover (SSC). SAC, was introduced and previously studied, is added here for the purpose of comparison and analysis. SSC extends SAC by more closely controlling the semantic distance between subtrees to which crossover may be applied. The new operators were tested on some real-valued symbolic regression problems and compared with standard crossover (SC), context aware crossover (CAC), Soft Brood Selection (SBS), and No Same Mate (NSM) selection. The experimental results show on the problems examined that, with computational effort measured by the number of function node evaluations, only SSC and SBS were significantly better
\end{abstract}

\footnotetext{
N. Q. Uy · M. O’Neill · E. Galván-López

Complex \& Adaptive Systems Lab, School of Computer Science \& Informatics,

University College Dublin, Dublin, Ireland

e-mail: quanguyhn@gmail.com

M. O’Neill

e-mail: m.oneill@ucd.ie

E. Galván-López

e-mail: edgar.galvan@ucd.ie

N. X. Hoai ( $\square)$

Department of Computer Science, Le Quy Don University, Hanoi, Vietnam e-mail: nxhoai@gmail.com

R. I. McKay

School of Computer Science and Engineering, Seoul National University,

Seoul, Korea

e-mail: rimsnucse@gmail.com
} 
than SC, and SSC was often better than SBS. Further experiments were also conducted to analyse the perfomance sensitivity to the parameter settings for SSC. This analysis leads to a conclusion that SSC is more constructive and has higher locality than SAC, NSM and SC; we believe these are the main reasons for the improved performance of SSC.

Keywords Genetic programming - Semantics - Crossover ·

Symbolic regression locality

\section{Introduction}

Genetic programming (GP) $[40,49,36])$ researchers have only recently paid much attention to semantic information, which has resulted in a dramatic increase in the number of realated publications (e.g. [26, 28, 29, 32, 31, 4, 43, 57, 10]). Previously, work in GP representation had focused mainly on syntactic aspects. From a programmer's perspective, however, maintaining syntactic correctness is only part of program construction: programs must be not only syntactically correct, but also semantically correct. Thus incorporating semantic awareness in the GP process could improve performance, extending the applicability of GP to problems that are difficult with purely syntactic approaches. So far, semantics has been incorporated into different phases of GP including fitness measurement [26, 29], operators execution [4, 57], valid checking [58, 33] and so forth. In this work, we investigate one method to incorporate semantic information into GP crossover operators for real-valued symbolic regression problems.

Previous evolutionary computation research has shown that characteristics of evolutionary operators such as their constructiveness, locality (small changes in genotype resulting in small changes in phenotype), and effect on population diversity strongly affect the performance of the resulting algorithms [8, 53, 51, 42]. However designing GP operators with these desirable characteristics can be very difficult. We aim to incorporate semantics into the design of new crossover operators so as to maintain greater semantic diversity, and provide greater constructiveness as well as higher locality than standard crossover (SC). We investigate the effects of these semantically-based operators on the performance of GP.

This paper addresses two main objectives. The first and narrower is to propose a new semantically-based schema for implementing crossover in GP that extends semantics aware crossover (SAC) in our previous work [57]. The second and broader objective is to encourage GP researchers to pay greater attention to the use of semantics to improve the efficiency of GP search. It extends [57] in a number of ways. First, we change the way the semantics is used to constrain the crossover, resulting in a new crossover that we call semantic similarity-based crossover (SSC). SSC extends SAC by not only encouraging exchange of semantically different material between parents, but also limiting this to small and controllable changes. SSC and SAC are compared with a broader class of related crossover operators in the literature and the results are positive. 
Experiments to investigate the impact of crucial parameters on SSC's performance are also presented, providing the basis on which to choose appropriate values for these parameters. Subsequently, we conduct a more comprehensive analysis to investigate the possible reasons behind the effectiveness of SSC, and in particular, why SSC works so much better than SC, SAC and NSM. Finally, we extend the previous work by comparison on a much broader range of target functions. All of these extensions will be presented in detail in the following sections.

The remainder of the paper is organised as follows. In the next section we review the literature on GP with semantic information and on GP crossover operators. Section 3 contains the detailed descriptions of our new crossovers. The experimental settings are described in Sect. 4. A comparison of the effectiveness of SSC and other related crossover operators are presented in Sect. 5. An analysis of parameter sensitivity for SSC and an investigation of some of the characteristics of SSC follow in the next two sections. The conclusions are presented in Sect. 8, leading to suggestions for future research in Sect. 9.

\section{Background}

In this section, we briefly review previous work on semantics in GP and on variants of GP crossover operators.

\subsection{Semantics in genetic programming}

Semantics is a broad concept that has been studied in a number of fields including Natural Language [1], Psychology [11] and Computer Science [46] among others. While the precise meaning varies from field to field, semantics is generally contrasted with syntax: the syntax refers to the surface form of an expression, while the semantics refers to its deeper meaning in some external worlds. In computer science, these external worlds are generally provided by their computational models.

In computer science, semantics can be informally defined as the meaning of syntactically correct programs or functions. Two programs that are syntactically the same must have the same semantics, but the converse may not be true.

As a simple example, consider two small programs shown in Eqs. 1 and 2. Syntactically, the first statement of each is identical, but the second statements differ. Semantically, however, they are identical: both programs compute the same result.

$$
\begin{aligned}
& x=1 ; \quad y=x+x ; \\
& x=1 ; \quad y=2 * x ;
\end{aligned}
$$

In GP, semantics has generally been used to provide additional guidance to the GP search. The necessary additional information is either added to or extracted from the GP individual's representation. Thus the available possibilities depend on the problem domain (Boolean or real-value,...), the GP individual representation (Grammar-, Tree- or Graph-based), and the search algorithm characteristics (fitness measure, genetic operators,...). 
To date, there have been three main approaches to representing and extracting semantics and using it to guide the evolutionary process:

1. grammar-based $[58,59,9,10,13,50]$

2. formal methods [26, 28, 29, 33, 32, 31]

3. GP s-tree representation [4, 6, 5, 43, 57, 37, 38]

The most popular form of the first uses attribute grammars. Attribute grammars are extensions of context-free grammars, in which a finite set of attributes provide context sensitivity [34]. GP individuals expressed in the form of attribute grammar trees can incorporate semantic information, which can be used to eliminate bad individuals (i.e., less fit individuals) from the population [13] or to prevent generating semantically invalid individuals as in [58, 59, 50, 9, 10]. The attributes used to present semantics are generally problem-dependent, and it is not always obvious how to determine the attributes for a given problem.

Recently, Johnson has advocated formal methods as a means to incorporate semantic information into the GP evolutionary process [26, 28, 29]. Formal methods are a class of mathematically-based techniques for the specification, development and verification of software and hardware systems [45]. They support the extraction and approximation of mathematical statements useful for system design and verification. In Johnson's work, semantic information extracted through formal methods, such as abstract interpretation or model checking, is used to quantify the fitness of individuals on some problems for which traditional sample-point-based fitness measure are unavailable or misleading. In [26, 28], Johnson used interval analysis (a form of abstract interpretation) to measure the fitness of individuals in solving a rectangle replacement problem and in robot control. By contrast, Keijzer [33] used interval analysis to check whether an individual is defined over the whole range of input values - if an individual is undefined anywhere, that individual can be assigned minimal fitness or simply deleted from the population. This allowed Keijzer to avoid discontinuities arising from protected operators, improving the evolvability of the system. Johnson [29] used model checking to measure individual fitness in evolving vending machine controllers. A controller is specified by a number of computation tree logic formulas [3]. Fitness of an individual is the number of formulas it satisfies. Subsequently, Katz and Peled [32, 31] also used model checking to define fitness in a GP system for the mutual exclusion problem. The advantage of formal methods lies in their strict mathematical background, potentially helping GP to evolve computer programs. However they are also high in complexity and difficult to implement, possibly explaining the limited research despite the advocacy of Johnson [27]. Their main application to date has lain in evolving control strategies.

Methods for extracting semantics from expression trees depend strongly on the problem domain. The finite inputs of Boolean domains mean that semantics can be accurately estimated in a variety of ways. Beadle and Johnson [4] investigated the effects of directly using semantic information to guide GP crossover on Boolean problem domains. They checked semantic equivalence between offspring and parents by transforming them to Reduced Ordered Binary Decision Diagrams 
(ROBDDs) [7]. Two trees are semantically equivalent if and only if they reduce to the same ROBDD. This is used to determine which participating individuals are copied to the next generation. If the offspring are semantically equivalent to their parents, the children are discarded and the crossover is restarted. This process is repeated until semantically new children are found. The authors argue that this results in increased semantic diversity in the evolving population, and a consequent improvement in the GP performance. This method of semantic equivalence checking is also applied to drive mutation [6] and guide the initialisation phase of GP [5], where the authors show that it benefits for GP in both phases. By contrast, McPhee et al. [43] extract semantic information from a Boolean expression tree by enumerating all possible inputs. They consider the semantics of two components in each tree: semantics of subtrees and semantics of context (the remainder of an individual after removing a subtree). They experimentally measured the variation of these semantic components throughout the GP evolutionary process. They paid special attention to fixed-semantic subtrees: subtrees where the semantics of the tree does not change when this subtree is replaced by another subtree. They showed that there may be very many such fixed semantic subtrees when the tree size increases during evolution; thus it becomes very difficult to change the semantics of trees with crossover and mutation once the trees have become large.

To the best of our knowledge, there has been no previous research on semantic guidance in real-valued problems before our own previous study [57]. There, we proposed a new crossover operator, SAC, based on checking the semantic equivalence of subtrees. SAC was tested on a family of real-valued symbolic regression problems, and was empirically shown to improve GP performance. SAC differs from Beadle and Johnson's approach [4] in two ways. First, the test domain is real-valued rather than Boolean. For real domains, it is not generally feasible to check semantic equivalence by reduction to a canonical form like a ROBDD. Second, the crossover operator is guided not by the semantics of the whole program tree, but by that of subtrees. This is inspired by recent work presented in [43] for calculating subtree semantics. However, for real domains, measuring semantics by enumerating all possible inputs as in [43] is also infeasible, so that the semantics must be approximated.

Recently, Krawiec and Lichocki proposed a way to measure the semantics of an individual based on fitness cases [37]. In this work, the semantics of an individual is defined as a vector in which each element is the output of the individual at the corresponding input fitness case. This semantics is used to guide crossover in a method similar to SBS, known as Approximating Geometric Crossover (AGC). In AGC, a number of children are generated at each crossover, the children most similar to their parents - in terms of semantics - being added to the next generation. The experiment is conducted on both real-valued and boolean regression problems. The results show that AGC is no better than SC in real-valued problems, and only slightly superior to SC in boolean problems. The same kind of semantics is then used to build functional modulation for GP [38], for which the experimental results show that it may be useful in characterising the compositionality and difficulty of a problem, potentially leading to performance improvements for GP. 


\subsection{Alternative crossovers in genetic programming}

It is well-known that crossover is the primary operator in GP [35]. In the standard crossover (SC) two parents are selected, and then one subtree is randomly selected in each parent. A procedure is called to check if these two subtrees are legal for crossover (syntactic closure properties, depth of resulting children,...). If so, the crossover is executed by simply swapping the two chosen subtrees, and the resulting offspring are added to the next generation. Figure 1 shows how SC works.

Much research has concentrated on the efficiency of crossover, resulting in new and improved operators which can be classified into three categories. These are:

1. crossovers based on syntax (structure)

2. crossovers based on context

3. crossovers based on semantics

Most of the early modifications to SC were based on syntax [35, 48, 39, 47, 24]. Koza [35] proposed a crossover that is $90 \%$ biased to function nodes and $10 \%$ bias to terminal nodes as crossover points. Although this method encourages the exchange of more genetic material (bigger subtrees) between the two participating individuals, it risks exacerbating bloat and thus making it more difficult to refine solutions in later generations [4]. O'Reilly and Oppacher [47] introduced height-fair crossover, in which all subtree heights in the two parents are recorded, and one subtree height is randomly selected. The crossover sites in both parents are then restricted to that particular height. Ito et al. [24] presented a similar depthdependent crossover, aiming to preserve building blocks. In this method, the probability of selecting a node is biased towards the root-nodes near the root have a greater probability to be selected for crossover. The bias of the selection

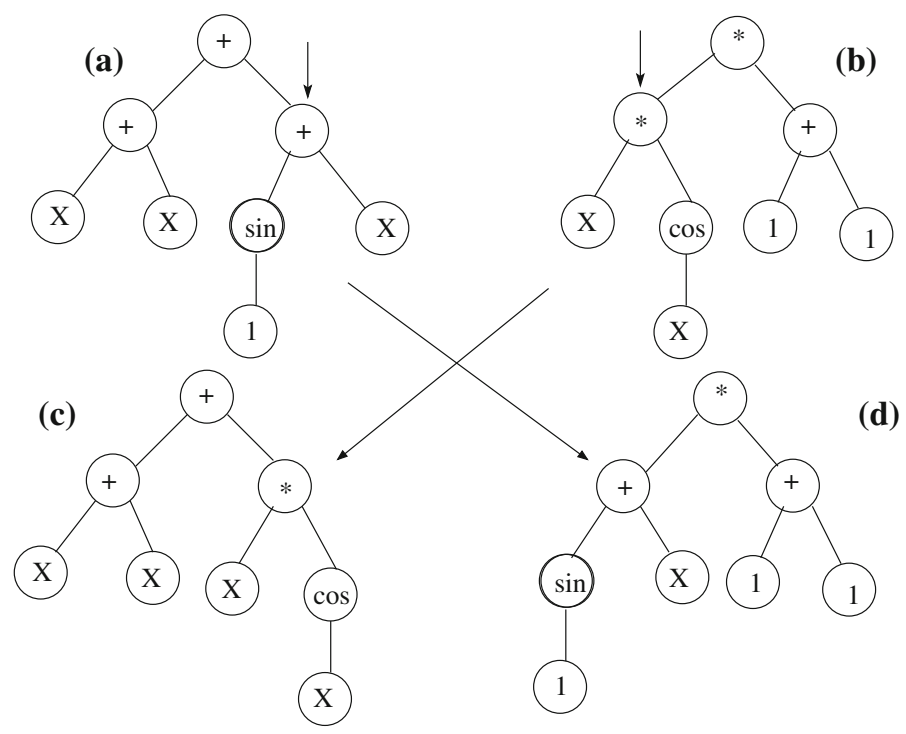

Fig. 1 Standard Crossover: parents (top) and their resulting offspring after applying crossover (bottom) 
probability is set by the user, and it is left unchanged during the search process. However it is not robust: if it is not carefully set for a particular problem, the performance can be very poor [25]. In [48, 39], Poli and Langdon introduced onepoint crossover and uniform crossover. In these methods, when two parents are selected for crossover, they are aligned based on their shapes. By aligning two parents, the common shape of these parents (starting from the roots) can be determined. The crossover points are then randomly selected from the nodes that lie in the common shape. This kind of crossover has been shown especially effective on Boolean problems as it causes a bigger genetic material exchange in earlier generations (in these generations the common shape is often very small) and yet can tune the solutions in later generations (when the common shapes are bigger).

More recently, context has been used as extra information for the selection of crossover points [2, 19, 41, 55, 56]. This class of crossover is perhaps closest to semantic based crossovers. Altenberg [2] proposed a new crossover inspired by the observation that in most animal species, breeding occurs more often than the number of surviving offspring might suggest. In other words, viable offspring are not always produced as a consequence of breeding. This crossover is called a Soft Brood Selection (SBS). Two parents are selected for crossover, then $N$ random crossovers are performed to generate a Brood of $2 N$ children. The children are evaluated and sorted based on their fitness. The two best children are copied to the next generation, the rest being discarded. This crossover was then developed by Tackett $[55,56]$ by using a subset of fitness cases to figure out which children in the Brood are added to the next generation. Hengpraprohm and Chongstitvatana [19] proposed Selective Crossover, in which each subtree is assigned an impact value, reflecting how well (or badly) the subtree affects the containing tree. The impact of a subtree is determined by removing that subtree and replacing it with a random terminal node. The change in resulting fitness is the impact value. The crossover is performed by replacing the worst subtree of each parent with the best subtree of the other. Majeed and Ryan [41] proposed Context Aware Crossover (CAC); after two parents have been selected for crossover, one subtree is randomly chosen in the first. This subtree is then crossed over into all possible locations in the second, all generated children being evaluated. The best child (based on fitness) is selected as the result, and copied to the next generation. The advantage of these context-based crossovers is increased probability of producing better children. On the other hand, it can be very time consuming to evaluate the context of each subtree.

To the best of our knowledge, the only previous use of semantics in crossover are those previously discussed in Sect. 2.1. They include Beadle and Johnson's [4] Semantics Driven Crossover for Boolean problems, Krawiec and Lichocki's [37] Approximating geometric crossover, and our previous work [57] on SAC.

\section{Methods}

In this section we give a detailed discussion of our two crossovers. We start by briefly describing how we measure semantics in real-valued problems. This allows us to define a concept of semantic distance, on the basis of which we propose two semantic relationships, which we then use to define two new crossover operators. 


\subsection{Measuring semantics}

As discussed in Sect. 2, the appropriate definition of semantics for GP is far from obvious. The semantics of an individual is often understood as the behavior of that individual with respect to a set of input values. However the possibilities for computing such semantics depends on the domain. For real-valued problems, both canonical-form methods corresponding to Beadle and Johnson's [4] Boolean ROBDDs, and complete enumeration as in McPhee's approach [43], are infeasible. Instead, we propose a simple way to estimate the semantics of subtrees, in which the semantics is approximated by evaluating the subtree on a pre-specified set of points in the problem domain. We call this Sampling Semantics. Formally, the Sampling Semantics of any tree (subtree) is defined as follows:

Let $F$ be a function expressed by a tree (subtree) $T$ on a domain $D$. Let $P$ be a set of points from domain $D, P=\left\{p_{1}, p_{2}, \ldots, p_{N}\right\}$. Then the Sampling Semantics of $T$ on $P$ in domain $D$ is the set $S=\left\{s_{1}, s_{2}, \ldots, s_{N}\right\}$ where $s_{i}=F\left(p_{i}\right), i=1,2, \ldots, N$.

For example, suppose that we are considering the interval $[0,1]$ and using a set of three points, $P=\{0,0.5,1\}$, for evaluating semantics. Then the Sampling Semantics of subtree $S t$ in Fig. 2 on $P$ is the set of three values $S=\{\sin (1)$ $-0, \sin (1)-0.5, \sin (1)-1\}=\{084,0.34,-0.16\}$. The value of $N$ depends on the problems. If it is too small, the approximate semantics might be too coarsegrained and not sufficiently accurate. If $N$ is too big, the approximate semantics might be more accurate, but more time consuming to measure. The choice of $P$ is also important. If the members of $P$ are too closely related to the GP function set (for example, $\pi$ for trigonometric functions, or $e$ for exponential/logarithmic functions), then the semantics might be misleading. For this reason, choosing them randomly may be the best solution. In this paper, the number of points for evaluating Sampling Semantics is set as the number of fitness cases for the problem (20 points for single variable functions and 100 points for bivariate functions, see Sect. 4), and we choose the set of points $P$ uniformly randomly from the problem domain. ${ }^{1}$

Based on the Sampling Semantics(SS), we define a Sampling Semantics Distance between two subtrees. In our previous work [57], we defined the Sampling Semantics Distance as the sum of the absolute differences for all values of SS. That is, let $P=\left\{p_{1}, p_{2}, \ldots, p_{N}\right\}$ and $Q=\left\{q_{1}, q_{2}, \ldots, q_{N}\right\}$ be the SS of Subtree $_{1}\left(\operatorname{St}_{1}\right)$ and Subtree $_{2}\left(S t_{2}\right)$ on the same set of sample points, then the Sampling Semantics Distance (SSD) between $S t_{1}$ and $S t_{2}$ was defined as:

$$
\operatorname{SSD}\left(S t_{1}, S t_{2}\right)=\left|p_{1}-q_{1}\right|+\left|p_{2}-q_{2}\right|+\cdots+\left|p_{N}-q_{N}\right|
$$

While the experiments in [57] showed that this SSD is beneficial, it has the undoubted weakness that the value of the SSD depends on the number of SS points $N$. To reduce this drawback, we now use the mean of the absolute differences as the SSD between subtrees. In other word, the SSD between $S t_{1}$ and $S t_{2}$ is defined as:

$$
\operatorname{SSD}\left(S t_{1}, S t_{2}\right)=\left(\left|p_{1}-q_{1}\right|+\left|p_{2}-q_{2}\right|+\cdots+\left|p_{N}-q_{N}\right|\right) / N
$$

\footnotetext{
1 Since Sampling Semantics is defined for any subtree, it can be used in particular to estimate the semantics of the whole tree. We will use it in this way in the examples in later sections.
} 


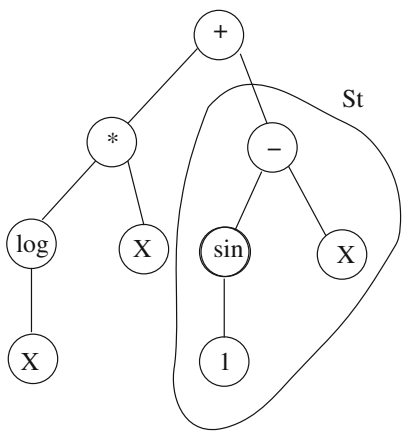

Fig. 2 Tree with subtree (for illustrating Sampling Semantics)

\subsection{Semantic relationships}

Based on Sampling Semantics Distance, we can define two semantic relationships between subtrees. Two subtrees are Semantically Equivalent (SE) on a domain if their SSD on the sample set of points is sufficiently similar (subject to a parameter called semantic sensitivity)—formally:

$$
\begin{aligned}
\mathrm{SE}\left(S t_{1}, S t_{2}\right)= & \text { if } \operatorname{SSD}\left(S t_{1}, S t_{2}\right)<\epsilon \text { then true } \\
& \text { else false }
\end{aligned}
$$

$\epsilon$ is the predefined semantic sensitivity. This subtree semantic relationship is similar to the metric we used in [57], and was inspired by the work of Mori et al. [44] on GP simplification. The experimental results in [57, 44] show that this semantic relationship benefits the GP search process.

The second relationship is known as Semantic Similarity. ${ }^{2}$ The intuition behind semantic similarity is that exchange of subtrees is most likely to be beneficial if the two subtrees are not semantically identical, but also not too semantically dissimilar. Two subtrees $S t_{1}$ and $S t_{2}$ are semantically similar on a domain if their SSD on the sample set lies within a positive interval-formally:

$$
\begin{aligned}
\mathrm{SSi}\left(S t_{1}, S t_{2}\right)= & \text { if } \alpha<\operatorname{SSD}\left(S t_{1}, S t_{2}\right)<\beta \text { then true } \\
& \text { else false }
\end{aligned}
$$

here $\alpha$ and $\beta$ are two predefined constants, known as the lower and upper bounds for semantic sensitivity, respectively. Conceivably, the best values for lower and upper bound semantic sensitivity might be problem dependent. However we suspect that for most symbolic regression problems, there is a wide range of appropriate values (see Sect. 6 , where we study various ranges of both lower and upper bound semantic sensitivity).

We note that there is some biological motivation for this approach. In mammals, the Major Histocompatibility Complex (MHC) genes (on chromosome 6 in humans)

\footnotetext{
${ }^{2}$ We are using similarity here in its ordinary English meaning, where A is similar to B implies that A is not the same as B, as opposed to a common mathematical convention in which similarity includes equivalence.
} 
play a major role in the immune response, and thus are a key part of our defences against disease, and subject to strong and rapidly-changing evolutionary pressures. However they also play an important role both in mate selection (partners in the same species, but with dissimilar MHC genes, are preferred), and in speciation, because differences in MHC that are too big may cause an immune response from the mother to the foetus. Thus in this case at least, biology also appears to favour crossovers with semantic similarity lying in a specific range.

We conclude this section by highlighting some important differences between our semantic relations and fitness. First, for fitness calculation we need to know the fitness cases, and fitness reflects how good (close to the target function) an individual is. In measuring SS, we do not need to know the fitness cases (of course semantics can be measured using the fitness cases, but different cases can also be used). Second, fitness is measured for the whole individual, while SS is mainly used to encapsulate the semantics of subtrees. The last and most important difference is the objective: fitness is used for individual selection while SS is used to guide crossover. It is also noted that the semantic definition in Krawiec and Lichocki [37] is a particular case of Sampling Semantics, in which the set of sample points is the the same as the set of fitness cases, and the semantics of the whole tree (a particular subtree) is used in crossover.

\subsection{Semantics aware crossover}

A SAC was first proposed in [57]. SAC is motivated by the observation that GP crossover may exchange semantically equivalent subtrees, resulting in children that are identical to their parents. Consider the two selected parents $P_{1}$ and $P_{2}$ shown at the top of Fig. 3. $P_{1}$ has the semantics $\sin (X)+3 X$ and $P_{2}$ has the semantics $4 X$. Subtree Sf $_{1} P_{1}$ and Subtree Sf $_{2} P_{2}$ are semantically equivalent subtrees, both having semantics $2 X$, although their structures are totally different. When these two subtrees are selected for crossover, the children are as shown in Fig. 4. Obviously, these two children have different syntax (structure) from, but identical semantics to, their parents. $C_{1}$ has semantics of $\sin (X)+3 X$ and $C_{2}$ has semantics of $4 X$. This leaves the fitness of the children unchanged after crossover.

SAC prevents the swapping of such semantically equivalent subtrees in crossover. Each time two subtrees are chosen for crossover, a semantic check
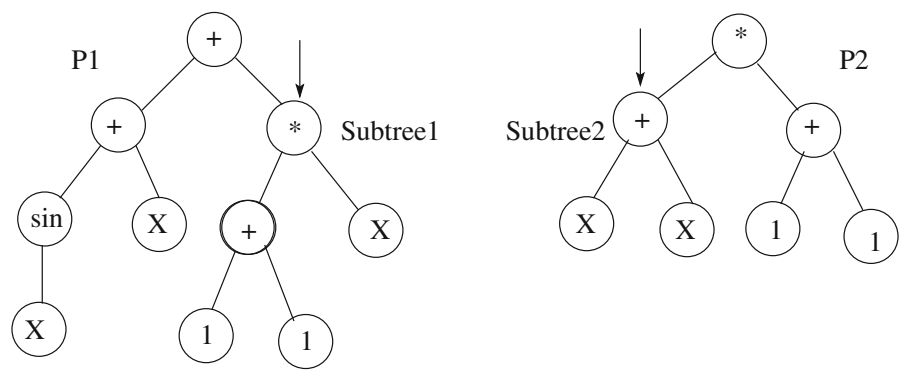

Fig. 3 Semantics equivalent subtrees are selected 

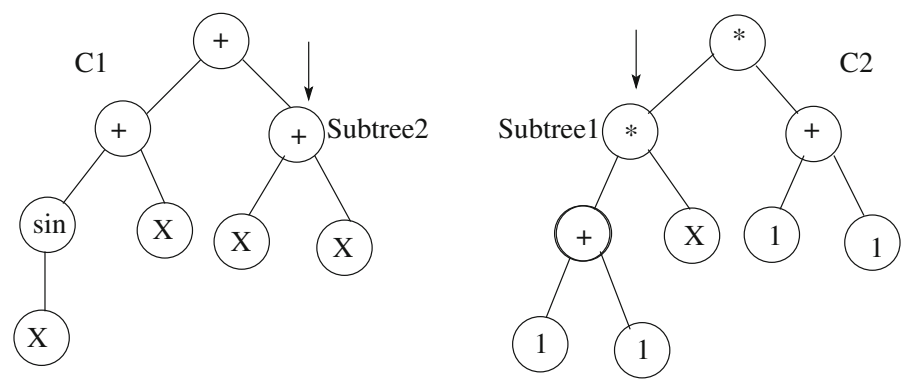

Fig. 4 The generated children from semantic equivalent subtree crossover

(using Semantic Equivalence) is performed to determine if they are equivalent. If they are, the crossover is aborted and instead performed on two other randomly chosen subtrees. Further detail on SAC can be found in [57]. SAC was partly inspired by Gustafson's No Same Mate selection [18] in which no two individuals with the same fitness may be selected for crossover. It, in turn, was motivated by experiments, in which he found that two parents with the same fitness often produce children with unchanged fitness upon crossover.

\subsection{Semantic similarity-based crossover}

The new semantically based crossover, SSC, is an extension of SAC in two ways. First, when two subtrees are selected for crossover, their semantic similarity, rather than semantic equivalence, is checked. Second, semantic similarity is more difficult to satisfy than semantic equivalence, so repeated failures may occur. As a result, SSC uses multiple trials to find a semantically similar pair, only reverting to random

Algorithm 1 Semantic similarity based crossover

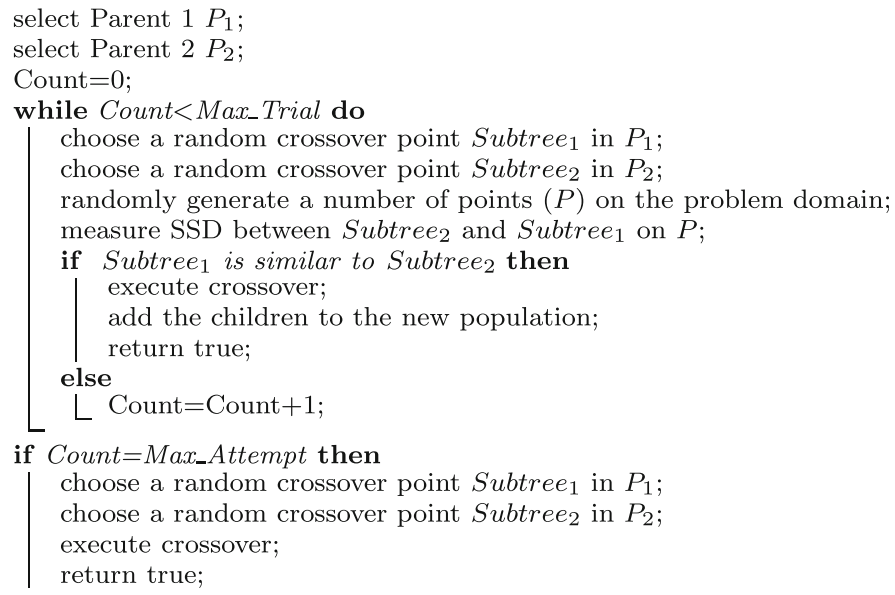


selection after passing a bound on the number of trials. Algorithm 1 shows how SSC works in detail.

In our experiments, we test a range of values of Max_Trial to gain an understanding of its effect on SSC. The motivation for SSC is to encourage exchange of semantically different, but not wildly different, subtrees. While forcing a change in the semantics of the individuals in the population, we want to keep this change bounded and small. We anticipate that a smoother change in semantics of the individuals will result, and might lead to a smoother change in fitness of the individuals after crossover. For instance, consider two parents selected for crossover in Fig. 5. Assume that we measure the SS of a tree on the 10 points, $P=\{1,2, \ldots, 10\}$. Then the SS of parents $P_{1}, P_{2}$ and of Subtree1 $\left(S t_{1}\right)$, Subtree2 $\left(S t_{2}\right)$, and Subtree3 $\left(S t_{3}\right)$ are as shown in Tables 1 and 2 . It can be seen from these tables that $S t_{1}$ and $S t_{2}$ are semantically similar (using $\alpha=10^{-4}, \beta=0.4$ as in this paper), with the SSD being only 0.09 , while $S t_{1}$ and $S t_{3}$ are semantically dissimilar since the SSD is 4.5. If crossover is performed by swapping two semantically similar subtrees $\left(S t_{1}\right.$ and $\left.S t_{2}\right)$, the generated children are show in Fig. 6. The SS of the two children $\left(C_{1}, C_{2}\right)$ are shown in Table 1 . We can also measure the SSD between $C_{1}$ and $P_{1}$ and between $C_{2}$ and $P_{2}$ (as shown in columns $C_{1}-P_{1}$ and $C_{2}-P_{2}$ in Table 1). Evidently, the change of semantics through crossover is quite small (1.1 with $\mathrm{C} 1$ and 1.65 with $\mathrm{C} 2$ ). This, we hope, will make for a smoother change of fitness.
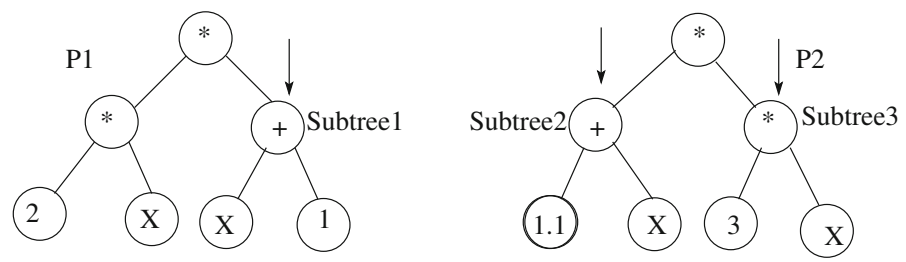

Fig. 5 Parents for crossover

Table 1 Sampling semantics of parents, subtrees and children when swapping two similar subtrees

\begin{tabular}{llrlrlllll}
\hline Points & $P_{1}$ & \multicolumn{1}{c}{$P_{2}$} & $S t_{1}$ & $S t_{2}$ & $S t_{1}-S t_{2}$ & $C_{1}$ & $C_{2}$ & $C_{1}-P_{1}$ & $C_{2}-P_{2}$ \\
\hline 1 & 4 & 6.3 & 2 & 2.1 & 0.1 & 4.2 & 6 & 0.2 & 0.3 \\
2 & 12 & 18.6 & 3 & 3.1 & 0.1 & 12.4 & 18 & 0.4 & 0.6 \\
3 & 24 & 36.9 & 4 & 4.1 & 0.1 & 24.6 & 36 & 0.6 & 0.9 \\
4 & 40 & 61.2 & 5 & 5.1 & 0.1 & 40.8 & 61 & 0.8 & 1.2 \\
5 & 60 & 91.5 & 6 & 6.1 & 0.1 & 61.0 & 91 & 1.0 & 1.5 \\
6 & 84 & 127.8 & 7 & 7.1 & 0.1 & 85.2 & 127 & 1.2 & 1.8 \\
7 & 112 & 170.1 & 8 & 8.1 & 0.1 & 113.4 & 170 & 1.4 & 2.1 \\
8 & 144 & 218.4 & 9 & 9.1 & 0.1 & 145.6 & 218 & 1.6 & 2.4 \\
9 & 180 & 272.7 & 10 & 10.1 & 0.1 & 181.8 & 272 & 1.8 & 2.7 \\
10 & 220 & 333.0 & 11 & 11.1 & 0.1 & 222.0 & 333 & 2.0 & 3.0 \\
SSD & & & & & 0.09 & & & 1.1 & 1.65 \\
\hline
\end{tabular}


Table 2 Sampling semantics of parents, subtrees and children when swapping two different subtrees

\begin{tabular}{llrllllllr}
\hline Points & $P_{1}$ & \multicolumn{1}{c}{$P_{2}$} & $S t_{1}$ & $S t_{3}$ & $S t_{1}-S t_{3}$ & $C_{3}$ & \multicolumn{1}{c}{$C_{4}$} & $C_{3}-P_{1}$ & $C_{4}-P_{2}$ \\
\hline 1 & 4 & 6.3 & 2 & 2 & 0 & 6 & 4.2 & 2 & 2.1 \\
2 & 12 & 18.6 & 3 & 4 & 1 & 24 & 9.3 & 12 & 9.3 \\
3 & 24 & 36.9 & 4 & 6 & 2 & 54 & 16.4 & 30 & 20.5 \\
4 & 40 & 61.2 & 5 & 8 & 3 & 96 & 25.5 & 56 & 35.7 \\
5 & 60 & 91.5 & 6 & 10 & 4 & 150 & 36.6 & 90 & 54.9 \\
6 & 84 & 127.8 & 7 & 12 & 5 & 216 & 49.7 & 132 & 78.1 \\
7 & 112 & 170.1 & 8 & 14 & 6 & 294 & 64.8 & 182 & 105.3 \\
8 & 144 & 218.4 & 9 & 16 & 7 & 384 & 81.8 & 240 & 136.5 \\
9 & 180 & 272.7 & 10 & 18 & 8 & 486 & 101.0 & 306 & 171.7 \\
10 & 220 & 333.0 & 11 & 20 & 9 & 600 & 122.1 & 380 & 201.0 \\
SSD & & & & & 4.5 & & & 143 & 82.5 \\
\hline
\end{tabular}
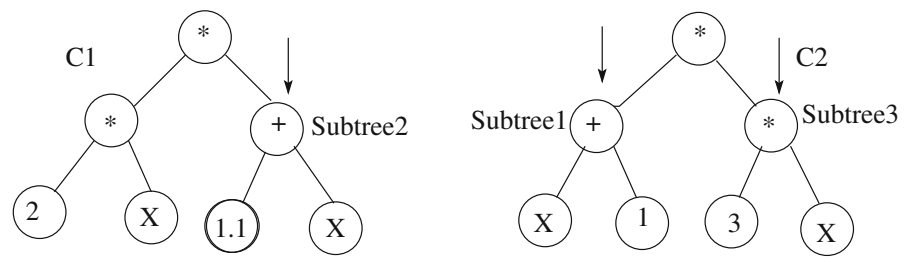

Fig. 6 Children generated by crossing over two semantically similar subtrees
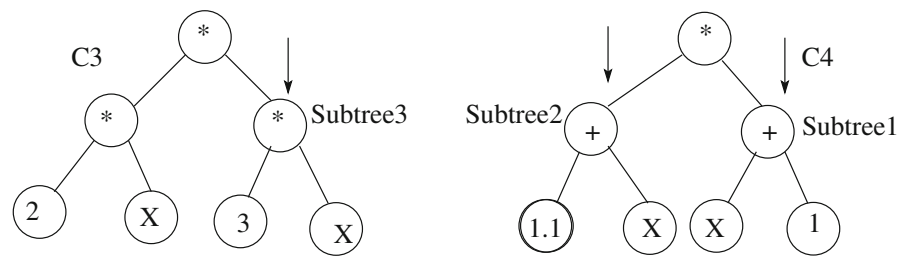

Fig. 7 Children generated by crossing over two semantically dissimilar subtrees

By contrast, if crossover is conducted by swapping two dissimilar subtrees $\left(S t_{1}\right.$ and $S t_{3}$ ), the children are shown in Fig. 7. The results of the calculation of the SS of the two children $\left(C_{3}\right.$ and $\left.C_{4}\right)$ and the semantic distances between these children and their parents are shown in Table 2. It can be seen from this table that the change in semantics between parents and children is rather large (143 and 82.5 for $C 3$ and $C 4$, respectively). This, we anticipate, will lead to an abrupt change in fitness after crossover.

\section{Experimental settings}

To experimentally investigate the possible effects of SSC in comparison with other crossover operators, we test them on ten real-valued symbolic regression problems. 
These problems are grouped into three categories: polynomial functions; trigonometric, logarithm and square-root functions; and bivariate functions. Most are taken from the works of Hoai et al. [20], Keijzer [33], and Johnson [30]. These functions are shown in Table 3 and the main parameters used for our experiments are given in Table 4. The parameter settings are similar to our previous work [57]. Although these experiments purely concern crossover, we have retained mutation at a low rate, because we aim to study crossover in the context of a normal GP run. Note that the number of generations and the population size are not specified in Table 4; they will be determined appropriately for each experiment. Note also that the raw fitness

Table 3 Symbolic regression functions

Table 4 Run and evolutionary parameter values

\begin{tabular}{ll}
\hline Functions & Fitcases \\
\hline$F_{1}=x^{3}+x^{2}+x$ & 20 random points $\subseteq[-1,1]$ \\
$F_{2}=x^{4}+x^{3}+x^{2}+x$ & 20 random points $\subseteq[-1,1]$ \\
$F_{3}=x^{5}+x^{4}+x^{3}+x^{2}+x$ & 20 random points $\subseteq[-1,1]$ \\
$F_{4}=x^{6}+x^{5}+x^{4}+x^{3}+x^{2}+x$ & 20 random points $\subseteq[-1,1]$ \\
$F_{5}=\sin \left(x^{2}\right) \cos (x)-1$ & 20 random points $\subseteq[-1,1]$ \\
$F_{6}=\sin (x)+\sin \left(x+x^{2}\right)$ & 20 random points $\subseteq[-1,1]$ \\
$F_{7}=\log (x+1)+\log \left(x^{2}+1\right)$ & 20 random points $\subseteq[0,2]$ \\
$F_{8}=\sqrt{x}$ & 20 random points $\subseteq[0,4]$ \\
$F_{9}=\sin (x)+\sin \left(y^{2}\right)$ & 100 random points $\subseteq[-1,1]$ \\
& $\mathrm{x}[-1,1]$ \\
$F_{10}=2 \sin (x) \cos (y)$ & 100 random points $\subseteq[-1,1]$ \\
& $\mathrm{x}[-1,1]$ \\
\hline
\end{tabular}

\begin{tabular}{ll}
\hline Parameter & Value \\
\hline Selection & Tournament \\
Tournament size & 3 \\
Crossover probability & 0.9 \\
Mutation probability & 0.05 \\
Initial max depth & 6 \\
Max depth & 15 \\
Max depth of mutation & 5 \\
tree & $+,-, *, /$ (protected versions), \\
Non-terminals & sin, cos, exp, log (protected versions) \\
Terminals & X, 1 for single variable problems, \\
& and X,Y for bivariable problems \\
Raw fitness & Sum of absolute error on all fitness \\
& cases \\
Hit & When an individual has an \\
absolute error $<0.01$ on a fitness case & When an individual scores hits \\
on all fitness cases & 100 independent runs for each value \\
Trials per treatment & \\
\hline &
\end{tabular}


function is the sum of the absolute error over all fitness cases, and a run is considered as successful when some individual hits (i.e. absolute error $<0.01$ ) every fitness case.

We divided our experiments into three sets. The first set investigates the performance of SSC. SSC was compared with five other crossover operators: Standard Crossover (SC), Semantics Aware Crossover (SAC), Context Aware Crossover (CAC), Soft Brood Selection (SBS), and No Same Mate (NSM) selection. The second set analyses the sensitivity of SSC's parameters-including lower and upper semantic sensitivities, maximum number of trials (Max_Trial), and number of sample points. The last set of experiments investigate some characteristics of SSC, including the rate of semantically equivalent crossover events, semantic diversity, locality, and constructivity. These three sets of experiments are detailed in the following sections.

\section{Comparative results}

This section presents our experimental results on the performance of SSC in comparison with SC, SAC, NSM, CAC, and SBS. When comparing different methods, one of the fundamental questions is how to compare their performance in a fair way. Traditionally, GP researchers often set up a predetermined population size and number of generations. Depending on the methods employed, the standard approach of comparing performance in terms of fitness at each generation may not be completely fair, due to possible differences in computational overhead.

An alternative, and often better, way is to run different GP systems (e.g., using different crossover methods) with the same predefined number of individual fitness evaluations. This would not, however, be fair in the context of this paper, because the semantic subtree checking in SSC and SAC may be performed on much smaller subtrees than the individuals (whole trees), and hence may cost much less. Moreover because of differences in bloat, the average size of the individuals in different methods may also differ [12, 22].

Here, we use a measure based on the number of function node evaluations to estimate the computational cost of each GP run. This kind of measurement has been adopted in a number of recent GP studies [23, 60]. By using the number of node evaluations, we can readily estimate the additional computational effort of nonstandard crossovers used in the experiments in this paper (i.e SAC, SSC, CAC, SBS). ${ }^{3}$ In these experiments, the number of node evaluations is set to $15^{*} 10^{6}$. This value was experimentally determined as allowing our base comparator, SC, to easily find solutions in the easy problems $\left(F_{1}\right)$, with about $50 \%$ successful runs, but not allowing it to readily find solutions to harder problems, with only about $5 \%$ success in $F_{4}$.

The experimental settings of these experiments were as follows. For all methods, the GP basic parameters were as in Table 4 . The population size for SC, SAC, SSC

\footnotetext{
${ }^{3}$ We assume that the computation costs of all primitive functions are the same, or at least negligibly different when compared to the cost of individual fitness evaluation.
} 
and NSM were set to 500 as in [57]. For SAC, the semantic sensitivity was set to $10^{-X}$ with $X=2,3,4$, and $5 .{ }^{4}$ For SSC, the lower and upper semantic sensitivity were set to $10^{-4}$ and 0.4 , respectively. The maximum number of attempts to form an SSC crossover Max_Trial was set to 8, 12, 16, and 20, forming four schemas of SSC. ${ }^{5}$ These values were determined from the experiments in Sect. 6, where they were found to be suitable values for the performances of SSC.

The population sizes we use for CAC and SBS follow previous research, where they are set much smaller than the population size for SC. Here, we chose 200 as in $[42,37]$. For CAC, we followed the Majeed and Ryan [42], in using CAC only after $80 \%$ of the node evaluations of a run. ${ }^{6} \mathrm{We}$ also extended CAC with a scheme similar to Tackett's [56], checking child fitness not only by using all fitness cases, but also through a subset of fitness cases. Ratios of $1 / X(X=1,2 \text {, and } 4)^{7}$ were used in this experiment (i.e. only $1 / X$ of the fitness cases were used to find the best of breed individual, reducing the overall cost).

For SBS [56], the original experiments used 4 brood sizes (2, 3, 4, 5). Here we used the best two $(3,4)$. To measure the fitness of the individuals in the brood, we used only a portion of the fitness cases, with a $1 / X(X=1,2,4)$ ratio. $^{8}$

To examine and compare the performance of these methods, we recorded two classic performance metrics, namely mean best fitness and the percentage of successful runs. The percentage of successful runs are recorded in Table 5, it should be noted here that a run is called successful run if it can find an individual that scores hits on all fitness cases, where a hit means that for that case, absolute error $<0.01$. In the resulapplied after $80 \%$ of individual evaluations for Methods. In each setting, the best-performing schema is printed in bold face. We can see that only SBS and SSC are definitely better than SC; while the performances of NSM and SAC are very similar to SC, CAC is often poorer. The reason might lie in the high cost of the method CAC uses to find the best crossover site, with the result that it quickly reaches the maximum function node evaluations, and the run terminates.

Turning specifically to SSC and SBS, we find that SSC is often better, and more consistently so, than SBS. While SSC is consistently superior on all tested functions, SBS seems to perform similarly to $\mathrm{SC}$ on some functions, such as $F_{1}, F_{2}$ and $F_{6}$. For SBS, reducing the number of fitness cases used to choose individuals from the brood improves the performance. It is not clear, however, to what extent we can reduce the number of fitness cases to further enhance the performance. In some cases, reducing only 2 times performs better than 4 times. For SSC, it seems that the values of Max_Trial from 8 to 20 give consistently good performance. In general, SSC performs better than SBS, and is the best of all methods on the the tested problems.

\footnotetext{
${ }^{4}$ SACs with different $X$ are denoted as SACX (with $X=2,3,4$, and 5).

5 Denoted as SSCX, where $X$ is $8,12,16$, and 20 .

${ }^{6}$ In the original version, $\mathrm{CAC}$ is applied after $80 \%$ of individual evaluations in a run. Here we use node evaluation for the purpose of uniform comparisons. We did, however, apply CAC earlier in the 40, 60\%, etc of the last node evaluations to compensate for this difference. The results were worse than those reported in the paper. We omit these results and only report the best results for CAC to compact the result tables.

7 Denoted as CACX with $X=1,2$, and 4 .

${ }^{8}$ Denoted as SBSXY, with $X=3,4$ and $Y=1,2,4$.
} 
Table 5 Number of successful runs out of 100 runs

\begin{tabular}{lllllllllll}
\hline Ms & F1 & F2 & F3 & F4 & F5 & F6 & F7 & F8 & F9 & F10 \\
\hline SC & 48 & 22 & 7 & 4 & 20 & 35 & 35 & 16 & 7 & 18 \\
NSM & 48 & 16 & 4 & 4 & 19 & 36 & 40 & 28 & 4 & 17 \\
SAC2 & 53 & 25 & 7 & 4 & 17 & 32 & 25 & 13 & 4 & 4 \\
SAC3 & 56 & 19 & 6 & 2 & 21 & 23 & 25 & 12 & 3 & 8 \\
SAC4 & 53 & 17 & 11 & 1 & 20 & 23 & 29 & 14 & 3 & 8 \\
SAC5 & 53 & 17 & 11 & 1 & 19 & 27 & 30 & 12 & 3 & 8 \\
CAC1 & 34 & 19 & 7 & 7 & 12 & 22 & 25 & 9 & 1 & 15 \\
CAC2 & 34 & 20 & 7 & 7 & 13 & 23 & 25 & 9 & 2 & 16 \\
CAC4 & 35 & 22 & 7 & 8 & 12 & 22 & 26 & 10 & 3 & 16 \\
SBS31 & 43 & 15 & 9 & 6 & 31 & 28 & 31 & 17 & 13 & 33 \\
SBS32 & 42 & 26 & 7 & 8 & 36 & 27 & 44 & 30 & 17 & 27 \\
SBS34 & 51 & 21 & 10 & 9 & 34 & 33 & 46 & 25 & 26 & 33 \\
SBS41 & 41 & 22 & 9 & 5 & 31 & 34 & 38 & 25 & 19 & 33 \\
SBS42 & 50 & 22 & 17 & 10 & 41 & 32 & 51 & 24 & 24 & 33 \\
SBS44 & 40 & 25 & 16 & 9 & 35 & 43 & 42 & 27 & 33 & 34 \\
SSC 8 & 66 & 28 & $\mathbf{2 2}$ & 10 & 48 & $\mathbf{5 6}$ & 59 & 21 & 25 & 47 \\
SSC12 & $\mathbf{6 7}$ & 33 & 14 & $\mathbf{1 2}$ & 47 & 47 & 66 & $\mathbf{3 8}$ & 37 & 51 \\
SSC16 & 55 & $\mathbf{3 9}$ & 20 & 11 & 46 & 44 & $\mathbf{6 7}$ & 29 & 30 & $\mathbf{5 9}$ \\
SSC20 & 58 & 27 & 10 & 9 & $\mathbf{5 2}$ & 48 & 63 & 26 & $\mathbf{3 9}$ & 51 \\
\hline
\end{tabular}

Table 6 shows the best fitness found, averaged over all 100 runs of each GP system. The results are consistent with those in Table 5, in that SAC and NSM are mostly equal to SC, CAC is often worse than SC, and only SBS and SSC are better than SC. The table again shows the consistently superior performance of SSC where it is better than SC on all test functions, while SBS is less convincing on three problems: $F_{1}, F_{2}, F_{6}$, and $F_{10}$. It can also be seen that although both SSC and SBS are superior to SC, the margin of improvement is different: SBS is often only slightly better than SC while SSC is widely better than SC in all cases.

We tested the statistical significance of the results in Table 6 using a Wilcoxon signed-rank test with a confidence level of $99 \%$. In Table 6 , if a run is significantly better than SC, its result is printed in italic face. It can be seen that while NSM is only significantly better than SC on one function $\left(F_{8}\right)$, SBS is regularly significantly better than SC, except on some specific functions, $F_{1}, F_{2}, F_{6}, F_{7}$, and $F_{10}$. SSC is always superior to SC in all cases and on all tested problems.

\section{SSC Parameter sensitivity analysis}

The experiments in this section investigate the effect of changing some parameters of SSC. The GP parameters were setup as in Table 4. The population size was set at 500 . Four parameters of SSC were investigated, namely, lower bound semantic sensitivity (LBSS), upper bound semantic sensitivity (UBSS), Max_Trial (MT), and the number of sample points (NP) used for semantic checking. First, we examined the effect of the 
Table 6 The mean best fitness of 100 runs

\begin{tabular}{|c|c|c|c|c|c|c|c|c|c|c|}
\hline Ms & $\mathrm{F} 1$ & $\mathrm{~F} 2$ & F3 & $\mathrm{F} 4$ & F5 & F6 & F7 & F8 & F9 & F10 \\
\hline $\mathrm{SC}$ & 0.18 & 0.26 & 0.39 & 0.41 & 0.21 & 0.22 & 0.13 & 0.26 & 5.54 & 2.26 \\
\hline NSM & 0.16 & 0.29 & 0.34 & 0.40 & 0.19 & 0.17 & 0.11 & 0.19 & 5.44 & 2.16 \\
\hline SAC2 & 0.16 & 0.27 & 0.42 & 0.50 & 0.22 & 0.23 & 0.15 & 0.27 & 5.99 & 3.19 \\
\hline SAC3 & 0.13 & 0.27 & 0.42 & 0.48 & 0.18 & 0.23 & 0.15 & 0.27 & 5.77 & 3.13 \\
\hline SAC4 & 0.15 & 0.29 & 0.41 & 0.46 & 0.17 & 0.22 & 0.15 & 0.26 & 5.77 & 3.03 \\
\hline SAC5 & 0.15 & 0.29 & 0.40 & 0.46 & 0.17 & 0.21 & 0.15 & 0.26 & 5.77 & 2.98 \\
\hline CAC1 & 0.33 & 0.41 & 0.51 & 0.53 & 0.31 & 0.42 & 0.17 & 0.35 & 7.83 & 4.40 \\
\hline CAC2 & 0.32 & 0.41 & 0.52 & 0.53 & 0.31 & 0.42 & 0.17 & 0.35 & 7.38 & 4.30 \\
\hline CAC4 & 0.33 & 0.41 & 0.53 & 0.53 & 0.30 & 0.42 & 0.17 & 0.35 & 7.80 & 4.32 \\
\hline SBS31 & 0.18 & 0.29 & 0.30 & 0.36 & 0.17 & 0.30 & 0.15 & 0.19 & 4.78 & 2.75 \\
\hline SBS32 & 0.18 & 0.23 & 0.28 & 0.36 & 0.13 & 0.28 & 0.10 & 0.18 & 4.47 & 2.77 \\
\hline SBS34 & 0.16 & 0.23 & 0.31 & 0.33 & 0.13 & 0.21 & 0.11 & 0.19 & 4.17 & 2.90 \\
\hline SBS41 & 0.18 & 0.26 & 0.27 & 0.38 & 0.12 & 0.20 & 0.13 & 0.20 & 4.40 & 2.75 \\
\hline SBS42 & 0.12 & 0.24 & 0.29 & 0.30 & 0.12 & 0.18 & 0.10 & 0.16 & 3.95 & 2.76 \\
\hline SBS44 & 0.18 & 0.24 & 0.33 & 0.35 & 0.15 & 0.16 & 0.11 & 0.19 & 2.85 & 1.75 \\
\hline SSC8 & 0.09 & 0.15 & 0.19 & 0.29 & 0.10 & 0.09 & 0.07 & 0.15 & 3.91 & 1.53 \\
\hline SSC12 & 0.07 & 0.17 & 0.18 & 0.28 & 0.10 & 0.12 & 0.07 & 0.13 & 3.54 & 1.45 \\
\hline SSC16 & 0.10 & 0.15 & 0.23 & 0.26 & 0.10 & 0.10 & 0.06 & 0.14 & 3.11 & 1.22 \\
\hline SSC 20 & 0.08 & 0.18 & 0.23 & 0.30 & 0.09 & 0.10 & 0.06 & 0.14 & 2.64 & 1.23 \\
\hline
\end{tabular}

A Wilcoxon signed-rank test was conducted; if a treatment is better than SC with a confidence level of $99 \%$, the result is printed in italic face

most important parameter, UBSS. We fixed the other parameters as follows: LBSS: $10^{-4}$, MT: 12 , and NP: 20 points for single variable functions and 100 for bivariate functions. The UBSS was set at 6 values: $0.1,0.2,0.4,0.6,0.8$, and $1 .{ }^{9}$

The second experiment analysed the effect of LBSS. In this experiment, the other parameters were set as follows: UBSS $=0.4, \mathrm{MT}=12$, and $\mathrm{NP}=$ number of fitness cases. Five values for LBSS were investigated, i.e. $10^{-X}$ where $\left(X=1,2,3,4\right.$, and 5). ${ }^{10}$

The third experiment tested sensitivity to the number of trials allowed in selecting similar subtrees in SSC (MT). For this experiment, LBSS $=10^{-4}$, $\mathrm{UBSS}=0.4$, and $\mathrm{NP}=$ number of fitness cases. MT was set at $4,8,12,16,20{ }^{11}$

The final experiment observed the effect of changing the number of sample points in semantic checking (NP). The experimental settings in this experiment were: $\mathrm{LBSS}=10^{-4}$, UBSS $=0.4$, and MT $=12$. NP was set to a ratio of $1 / 2,1$ or 2 of the number of fitness cases. ${ }^{12}$

To estimate the effect of changing these parameters, we recorded the best fitness of a run. These values were averaged over 100 runs, the results being shown in

\footnotetext{
9 Denoted as SSCUX where $X$ is $0.1,0.2,0.4,0.6,0.8$, or 1 .

${ }^{10}$ Denoted as SSCLX with $X=1,2,3,4$, and 5 .

11 Denoted as SSCMTX, with $X=4,8,12,16$, and 20.

12 Denoted as SSCNPX with $X=0.5,1$ or 2 .
} 
Table 7 The mean best fitness of 100 runs of SSC with different parameter values

\begin{tabular}{lllllllllll}
\hline Ms & F1 & F2 & F3 & F4 & F5 & F6 & F7 & F8 & F9 & F10 \\
\hline SSCU01 & 0.12 & 0.18 & 0.24 & 0.35 & 0.12 & 0.14 & 0.10 & 0.19 & 2.65 & 0.98 \\
SSCU02 & 0.09 & 0.17 & 0.20 & 0.30 & 0.09 & 0.10 & 0.08 & 0.15 & 1.95 & 0.83 \\
SSCU04 & 0.08 & 0.14 & 0.21 & 0.27 & 0.11 & 0.07 & 0.07 & 0.11 & 1.00 & 0.70 \\
SSCU06 & 0.06 & 0.16 & 0.22 & 0.28 & 0.11 & 0.07 & 0.08 & 0.12 & 2.01 & 0.68 \\
SSCU08 & 0.06 & 0.19 & 0.21 & 0.29 & 0.14 & 0.12 & 0.08 & 0.13 & 2.43 & 0.96 \\
SSCU1 & 0.09 & 0.19 & 0.26 & 0.31 & 0.16 & 0.15 & 0.10 & 0.15 & 2.53 & 1.26 \\
SSCL1 & 0.10 & 0.16 & 0.24 & 0.31 & 0.15 & 0.11 & 0.10 & 0.15 & 1.75 & 1.06 \\
SSCL2 & 0.06 & 0.14 & 0.22 & 0.27 & 0.10 & 0.07 & 0.07 & 0.13 & 1.32 & 0.66 \\
SSCL3 & 0.09 & 0.15 & 0.22 & 0.26 & 0.11 & 0.08 & 0.07 & 0.12 & 0.99 & 0.73 \\
SSCL4 & 0.08 & 0.14 & 0.21 & 0.27 & 0.12 & 0.07 & 0.07 & 0.11 & 1.00 & 0.70 \\
SSCL5 & 0.09 & 0.15 & 0.22 & 0.29 & 0.13 & 0.07 & 0.07 & 0.11 & 1.01 & 0.73 \\
SSCMT4 & 0.10 & 0.20 & 0.23 & 0.32 & 0.12 & 0.11 & 0.10 & 0.15 & 1.86 & 0.85 \\
SSCMT8 & 0.08 & 0.15 & 0.21 & 0.24 & 0.11 & 0.09 & 0.07 & 0.13 & 1.12 & 0.71 \\
SSCMT12 & 0.08 & 0.14 & 0.21 & 0.27 & 0.12 & 0.07 & 0.07 & 0.11 & 1.00 & 0.70 \\
SSCMT16 & 0.09 & 0.16 & 0.19 & 0.26 & 0.10 & 0.09 & 0.07 & 0.11 & 0.98 & 0.78 \\
SSCMT20 & 0.08 & 0.15 & 0.19 & 0.22 & 0.09 & 0.08 & 0.07 & 0.10 & 1.20 & 0.66 \\
SSCNP05 & 0.07 & 0.16 & 0.20 & 0.25 & 0.10 & 0.08 & 0.11 & 0.16 & 1.28 & 0.70 \\
SSCNP1 & 0.08 & 0.14 & 0.21 & 0.27 & 0.12 & 0.07 & 0.07 & 0.11 & 1.00 & 0.70 \\
SSCNP2 & 0.07 & 0.14 & 0.21 & 0.26 & 0.11 & 0.07 & 0.09 & 0.13 & 1.11 & 0.79 \\
\hline SSCUX & & & & & & & & &
\end{tabular}

SSCUX shows the effect of upper bound semantic sensitivity, SSCLX of lower bound semantic sensitivity, SSCMTX that of Max_Trial (X) and SSCNPX that of the number of sample points

Table 7. We can see that the value of UBSS has a remarkable effect on the performance of SSC. It seems that values from 0.2 to 0.8 are suitable for the problems under test, with values from 0.4 to 0.6 being the best. If UBSS is too small (0.1) or too big (1) the performance of SSC is poorer. This can be explained by recording the percentage of SSC that successfully selects two semantically similar subtrees, as shown in Table $8 .{ }^{13}$ We can see that if UBSS is too small, only a few SSCs can succeed in exchanging semantically similar subtrees (from 30 to $40 \%$ when UBSS is 0.1 ), so that SSC underperforms. ${ }^{14}$ By contrast, if UBSS is too large, it is almost trivial to find semantically similar subtrees (almost 100\% for UBSS $=1$ ) because most subtrees are sufficiently semantically similar, so that SSC behaves like SC.

While changing UBSS has a remarkable effect on SSC, LBSS has almost no effect on performance provided it is sufficiently small. Table 7 shows that while LBSS was set to small values (from $10^{-2}$ to $10^{-5}$ ), the performance of SSC was almost unchanged. In order to understand this, we recorded the percentage of subtrees with SSD smaller than $10^{-2}$ that are actually semantically identical. In fact,

\footnotetext{
13 The values for SSCLX and SSCPX are not shown in this table as they have little effect.

14 We have tried increasing the Max_Trial to compensate for decreasing the upper bound. This was unsuccessful, as if UBSS is too small, the exchange of semantics between the two parents is also too small, so that SSC is more readily trapped in local optima.
} 
Table 8 The percentage of SSC that successfully exchange two semantically similar subtrees

\begin{tabular}{lllllllllll}
\hline Ms & F1 & F2 & F3 & F4 & F5 & F6 & F7 & F8 & F9 & F10 \\
\hline SSCU01 & 42.1 & 40.9 & 45.6 & 42.2 & 39.6 & 49.3 & 25.2 & 27.9 & 39.8 & 46.4 \\
SSCU02 & 57.6 & 62.4 & 62.8 & 61.9 & 68.3 & 73.2 & 52.7 & 44.1 & 56.3 & 64.2 \\
SSCU04 & 77.2 & 81.1 & 79.4 & 78.4 & 81.2 & 85.4 & 81.2 & 67.3 & 74.8 & 80.5 \\
SSCU06 & 94.5 & 95.1 & 95.2 & 95.2 & 95.5 & 96.1 & 97.6 & 88.9 & 93.4 & 95.2 \\
SSCU08 & 97.2 & 98.4 & 98.3 & 98.3 & 98.5 & 97.7 & 99.3 & 95.4 & 96.4 & 98.5 \\
SSCU1 & 99.6 & 99.8 & 99.8 & 99.7 & 99.9 & 99.7 & 99.9 & 98.9 & 99.3 & 99.6 \\
SSCNP4 & 42.4 & 41.6 & 42.1 & 41.2 & 44.0 & 48.5 & 47.6 & 29.4 & 38.2 & 44.6 \\
SSCNP8 & 64.9 & 67.5 & 66.3 & 66.5 & 68.3 & 74.5 & 71.2 & 52.4 & 62.5 & 68.2 \\
SSCNP12 & 77.2 & 81.1 & 79.4 & 78.4 & 81.2 & 85.4 & 81.2 & 67.3 & 74.8 & 80.5 \\
SSCNP16 & 85.5 & 86.9 & 86.4 & 86.2 & 88.9 & 90.5 & 89.1 & 74.3 & 82.4 & 86.8 \\
SSCNP20 & 90.4 & 91.5 & 90.8 & 90.7 & 93.4 & 93.9 & 92.8 & 83.5 & 93.8 & 96.4 \\
\hline
\end{tabular}

99\% of such semantically equivalent subtrees actually have the same semantics. Thus $99 \%$ of these subtrees would have satisfied the equivalence condition regardless of the values of LBSS. Only in the case when LBSS gets too big, e.g. 0.1, does SSC have poorer performance. In this case, SSC prevents swapping of subtrees with similar but unequal semantics. We recorded how many subtree checks found a nonzero SSD smaller than 0.1 ; this happened approximately $20 \%$ of the time, misleading SSC. In general, we can see that LBSS is only required to be sufficiently small, and perhaps any value under $10^{-2}$ would be suitable.

The third parameter investigated is the number of unsuccessful trials permitted in selecting semantically similar subtrees (MT). Values of MT from 8 to 20 keep the performance of SSC roughly consistent. When MT is too small, e.g. MT $=4$, the performance of SSC is worse. This can also be understood by observing the percentage of SSC events that successfully exchanged two semantically similar subtrees. For MT $=4$, only 30 to $40 \%$ of SSC events successfully exchanged subtrees, while this figure rises to about $90 \%$ for MT $=20$. Thus further increasing MT may not help, because nearly all crossover events have already successfully exchanged semantically similar subtrees.

The last parameter under investigation is the number of sample points (NP) on which the semantics was measured. Usually, this number is set equal to the number of fitness cases. The results in Table 7 show that there was little effect when this value was doubled, or when it was halved.

Overall, these results highlight some important issues in determining the values for SSC parameters. It seems that UBSS should lie in the range 0.2 to 0.8 , LBSS should be less than $10^{-2}$, MT in the range 8 to 20 , and NP similar to the number of fitness cases so long as this number is not too big.

\section{Some characteristics of semantic similarity based crossover}

This section analyses some characteristics of SSC, namely the rate at which semantically equivalent crossover events occur, the semantic diversity resulting 
Table 9 The percentage of semantically equivalent crossover for four crossover operators: SC, NSM, SAC and SSC

\begin{tabular}{lllllllllll}
\hline Ms & F1 & F2 & F3 & F4 & F5 & F6 & F7 & F8 & F9 & F10 \\
\hline SC & 15.5 & 14.4 & 14.7 & 14.1 & 13.2 & 15.7 & 14.3 & 14.4 & 12.4 & 14.1 \\
NSM & 14.9 & 14.1 & 14.5 & 13.8 & 12.5 & 14.8 & 14.2 & 14.1 & 11.5 & 12.7 \\
SAC1 & 3.54 & 3.12 & 3.18 & 3.12 & 3.28 & 3.84 & 3.32 & 3.34 & 3.49 & 3.67 \\
SAC2 & 2.18 & 1.88 & 1.93 & 1.86 & 1.53 & 2.24 & 1.80 & 1.89 & 1.49 & 1.99 \\
SAC3 & 2.16 & 1.85 & 1.90 & 1.65 & 1.52 & 2.08 & 1.77 & 1.88 & 1.47 & 2.01 \\
SAC4 & 2.15 & 1.83 & 1.88 & 1.84 & 1.52 & 2.04 & 1.75 & 1.86 & 1.46 & 1.97 \\
SAC5 & 2.10 & 1.82 & 1.87 & 1.84 & 1.51 & 2.01 & 1.72 & 1.81 & 1.46 & 2.03 \\
SSC4 & 8.87 & 8.33 & 8.35 & 8.06 & 6.83 & 7.81 & 7.27 & 9.80 & 7.63 & 6.56 \\
SSC8 & 5.92 & 4.76 & 4.97 & 4.76 & 3.89 & 3.88 & 4.00 & 6.94 & 4.76 & 3.75 \\
SSC12 & 4.11 & 2.76 & 3.10 & 3.04 & 2.25 & 2.38 & 2.70 & 5.08 & 3.24 & 2.84 \\
SSC16 & 2.80 & 1.99 & 2.27 & 2.00 & 1.39 & 1.61 & 1.65 & 3.94 & 1.99 & 2.04 \\
SSC20 & 2.29 & 1.49 & 1.57 & 1.40 & 0.96 & 1.19 & 1.24 & 2.82 & 1.49 & 1.76 \\
\hline
\end{tabular}

from such crossovers, the locality of the operator, and its constructive effect. The results were compared with SC, SAC and NSM. The GP parameter settings in this section are described in Table 4, with the population size being set to 500 and the number of generations to 50. Five configurations of SAC were used, with semantic sensitivities set to $10^{-X}$ with $X=1,2,3,4$, and $5{ }^{15}$ For SSC, LBSS was set to $10^{-4}$ and UBSS to 0.4. NP was set equal to the number of fitness cases. Five configurations of SSC were used, with MT varying through 4, 8, 12, 16, and 20.

\subsection{Rates of semantically equivalent crossover events}

The first set of results record the extent of semantically equivalent exchanges arising from the four crossover operators. Here we say that a crossover operation is an equivalent crossover if it is performed by exchanging two semantically equivalent subtrees. Since the new crossover operators (SAC and SSC) work by checking the semantics of subtrees and trying to prevent the exchange of semantically equivalent subtrees, it would be informative to see how frequently this actually happens. This information shows us how frequently SC fails to change the semantics of individuals (i.e. makes semantically unproductive crossovers), and the extent to which SAC, and especially SSC, can overcome this problem. The results are shown in Table 9.

It can be seen from Table 9 that the overall average for equivalent crossovers in SC was around 15\%; NSM behaved similarly, only reducing the rate by about $1 \%$. By contrast, these values for both SAC and SSC were substantially improved, ranging from 2 to $3 \%$ for SAC, and from 2 to $5 \%$ for SSC (except when MT is very small, e,g MT = 4). It is clear that SAC and SSC are more semantically exploratory than SC and NSM on these problems. It should also be noted here that $99 \%$ of pairs

$\overline{15 \text { Denoted as SACX, for } X=1}, 2,3,4$, or 5 . 
Table 10 The percentage of generating new semantics for SC, NSM, SAC and SSC (i.e. differing in terms of the semantic equivalence measure)

\begin{tabular}{lllllllllll}
\hline Ms & F1 & F2 & F3 & F4 & F5 & F6 & F7 & F8 & F9 & F10 \\
\hline SC & 62.9 & 67.9 & 67.6 & 68.1 & 61.5 & 67.0 & 66.4 & 61.0 & 74.8 & 74.9 \\
NSM & 66.9 & 70.4 & 70.1 & 72.2 & 63.2 & 70.9 & 71.1 & 66.6 & 78.3 & 77.6 \\
SAC1 & 70.2 & 73.5 & 75.1 & 76.1 & 65.2 & 74.1 & 73.7 & 70.2 & 82.1 & 84.6 \\
SAC2 & 71.3 & 74.5 & 77.4 & 77.6 & 66.5 & 75.9 & 76.7 & 71.7 & 83.8 & 86.7 \\
SAC3 & 72.1 & 74.7 & 77.6 & 78.3 & 67.7 & 76.9 & 75.4 & 71.7 & 84.1 & 86.3 \\
SAC4 & 71.6 & 75.1 & 77.4 & 77.5 & 66.1 & 76.7 & 75.2 & 71.5 & 84.1 & 86.5 \\
SAC5 & 71.4 & 74.9 & 77.1 & 77.5 & 65.9 & 76.8 & 75.4 & 71.8 & 84.3 & 85.8 \\
SSC4 & 72.1 & 76.3 & 77.4 & 79.1 & 69.2 & 76.3 & 75.6 & 75.5 & 78.7 & 82.8 \\
SSC8 & 75.9 & 80.7 & 80.8 & 82.9 & 73.6 & 82.7 & 81.3 & 74.8 & 80.8 & 89.3 \\
SSC12 & 78.9 & 84.1 & 84.5 & 84.3 & 78.3 & 83.8 & 82.8 & 76.9 & 85.8 & 90.2 \\
SSC16 & 78.8 & 85.9 & 85.6 & 87.2 & 78.1 & 86.9 & 85.2 & 78.6 & 89.8 & 91.6 \\
SSC20 & 77.9 & 85.3 & 86.7 & 87.4 & 79.1 & 84.5 & 83.9 & 78.7 & 88.9 & 91.0 \\
\hline
\end{tabular}

of semantically equivalent subtrees consist of subtrees with identical semantics. As a result, approximately $99 \%$ of such crossovers leave the fitnesses of the children unchanged.

The improved semantic exploratory capacity of SAC and SSC can potentially lead to more semantic diversity, in that they could generate more new semantics than SC and NSM. Here, crossover A is considered to generate more semantic diversity than crossover B if A generates semantically new children, differing from the semantics of the parents, at a higher rate than B. In Table 10 we measured this rate for each crossover configuration. In Table 10 we see that while NSM was only slightly better than SC, SAC was better than both, while SSC was better than all other crossover operators in this respect. Interestingly, although SAC was often better than SSC in preventing equivalent crossovers, by keeping semantic changes small, SSC was generally better than SAC at producing semantically diverse crossovers. We note that SAC and SSC cannot guarantee the generation of semantically new offspring, despite trying to swap semantically different subtrees. We suspect this arises from the existence of fixed-semantic subtrees similar to those whose existence McPhee et al. demonstrated in Boolean domains [43].

\subsection{Operator locality}

The next set of experiments analyse the locality of SSC compared with SAC, SC and NSM. It is generally believed that using a representation with high locality (small change in genotype correspond to small change in phenotype) is important for efficient evolutionary search $[17,21,52,15,16]$. It is also generally agreed that designing a search operator for GP ensuring which achieves this is very difficult. Thus most current GP representations and operators are low-locality-a small (syntactic) change from parent to child can cause a large semantic change. Our new crossover operator (SSC) differs from others in directly controlling the scale of 
Table 11 The average change of fitness after crossover for SC, NSM, SAC, and SSC (averaged over the whole population and 100 runs)

\begin{tabular}{lllllllllll}
\hline Ms & F1 & F2 & F3 & F4 & F5 & F6 & F7 & F8 & F9 & F10 \\
\hline SC & 9.74 & 9.21 & 10.5 & 10.6 & 7.30 & 7.44 & 8.13 & 9.36 & 17.8 & 20.1 \\
NSM & 7.64 & 8.30 & 9.21 & 10.1 & 6.00 & 6.34 & 7.40 & 7.84 & 14.7 & 18.5 \\
SAC1 & 8.42 & 8.71 & 9.54 & 10.9 & 7.01 & 6.54 & 7.05 & 7.96 & 15.9 & 17.7 \\
SAC2 & 8.38 & 8.69 & 9.42 & 10.8 & 6.93 & 6.48 & 6.96 & 7.85 & 15.8 & 17.5 \\
SAC3 & 8.03 & 8.63 & 9.19 & 10.3 & 6.82 & 6.56 & 6.92 & 7.66 & 15.5 & 17.3 \\
SAC4 & 7.88 & 8.64 & 9.03 & 10.4 & 7.14 & 6.65 & 7.30 & 7.60 & 15.5 & 17.1 \\
SAC5 & 7.88 & 8.70 & 9.43 & 10.4 & 7.18 & 6.78 & 7.25 & 7.68 & 15.4 & 17.4 \\
SSC4 & 6.83 & 6.41 & 6.72 & 7.11 & 5.06 & 4.60 & 5.38 & 6.50 & 13.1 & 13.5 \\
SSC8 & 5.12 & 5.01 & 5.69 & 5.45 & 3.58 & 3.47 & 3.87 & 5.84 & 12.4 & 9.50 \\
SSC12 & 4.07 & 4.00 & 4.90 & 4.97 & 3.09 & 2.70 & 3.32 & 5.26 & 11.3 & 8.76 \\
SSC16 & 4.34 & 3.44 & 4.26 & 4.10 & 2.84 & 2.58 & 2.82 & 4.45 & 9.25 & 7.83 \\
SSC20 & 4.19 & 3.22 & 3.55 & 3.90 & 2.56 & 2.26 & 2.97 & 3.64 & 7.32 & 9.15 \\
\hline
\end{tabular}

change in terms of semantics rather than syntax. Moreover, the concept of locality used in SSC is slightly different from the literature in that while guaranteeing small changes in genotype only cause small changes in phenotype (by using an upper bound), SSC does use a lower bound to enforce the change in semantics.

To compare locality, we measured the fitness change between parents and children in crossover. For example, suppose two individuals having fitness of 10 and 15 are selected for crossover, and their children have fitness of 17 and 9, respectively. The change of fitness is $A b s(17-10)+A b s(9-15)=13$ (for this purpose, we compare the fitness of a child with that of the parent in which it is rooted). This value was averaged over the whole population and over 100 runs. The average fitness change of individuals before and after crossover is shown in Table 11.

Table 11 shows that the step size of the fitness change for SSC was much smaller than for either SAC, SC or NSM. This leads to smoother fitness change over time for SSC than for the others. This is important, as it is not easy to ensure the locality property in GP. The table also reveals that the fitness change in SAC and NSM were only slightly smoother than in SC.

\subsection{Constructive effects}

The previous results show that SAC and SSC are more semantically productive than SC and NSM, and that SSC has higher locality than the others. Does this help SSC (and maybe SAC) to generate better children than their parents (more constructive crossover)? We measured the constructive effect of SAC, SSC, NSM and SC, using Majeed's [42] method. However we adapt the method slightly, only comparing the fitness of a child with that of the parent in which it is rooted.

We can distinguish semi-constructive crossovers from full-constructive crossovers. Let us assume that two parents $P_{1}$ and $P_{2}$ are selected for crossover, 
Table 12 The percentage of semi-constructive crossovers of SC, NSM, SAC, and SSC (i.e. at least one child is better than the corresponding parent)

\begin{tabular}{lllllllllll}
\hline Ms & F1 & F2 & F3 & F4 & F5 & F6 & F7 & F8 & F9 & F10 \\
\hline SC & 19.2 & 21.2 & 21.0 & 21.1 & 18.7 & 21.1 & 21.4 & 18.6 & 28.2 & 28.9 \\
NSM & 22.2 & 23.3 & 22.5 & 23.8 & 19.5 & 23.8 & 24.1 & 21.5 & 31.6 & 30.6 \\
SAC1 & 24.7 & 26.3 & 26.5 & 26.7 & 22.3 & 26.1 & 27.2 & 24.2 & 34.1 & 34.2 \\
SAC2 & 25.4 & 26.3 & 27.8 & 27.8 & 23.5 & 27.5 & 28.8 & 25.6 & 36.0 & 36.9 \\
SAC3 & 25.9 & 26.5 & 27.8 & 28.3 & 23.8 & 28.2 & 27.6 & 25.5 & 36.2 & 36.7 \\
SAC4 & 25.7 & 27.0 & 27.7 & 27.8 & 23.2 & 28.2 & 27.4 & 25.5 & 36.4 & 37.4 \\
SAC5 & 25.7 & 26.9 & 27.6 & 27.9 & 22.9 & 28.6 & 27.5 & 25.6 & 36.2 & 37.1 \\
SSC4 & 26.9 & 28.2 & 29.0 & 29.3 & 25.7 & 28.9 & 28.7 & 25.9 & 33.4 & 36.8 \\
SSC8 & 30.0 & 32.1 & 32.0 & 32.7 & 29.9 & 33.9 & 33.2 & 29.0 & 36.8 & 41.2 \\
SSC12 & 32.7 & 35.5 & 34.6 & 34.3 & 32.9 & 35.5 & 35.2 & 31.9 & 38.5 & 43.1 \\
SSC16 & 33.3 & 37.1 & 35.9 & 37.1 & 33.6 & 37.2 & 36.3 & 34.1 & 41.5 & 43.3 \\
SSC20 & 32.7 & 36.7 & 37.0 & 36.9 & 34.7 & 36.0 & 36.0 & 34.0 & 41.4 & 42.8 \\
\hline
\end{tabular}

generating two children $C_{1}, C_{2}\left(C_{1}\right.$ rooted in $P_{2}$ and $C_{2}$ rooted in $\left.P_{2}\right)$. Then, a crossover is called semi-constructive if it generates at least one child that is better than its parents. In other words, the condition $\left(C_{1}\right.$ is better than $P_{1}$ OR $C_{2}$ is better than $P_{2}$ ) is used to count semi-constructive crossovers. When the condition is more strict-both children are better than their parents $\left(C_{1}\right.$ is better than $P_{1} A N D C_{2}$ is better than $P_{2}$ ) - the crossover is called full-constructive. A crossover that is not semi-constructive nor full-constructive is called destructive.

The semi-constructive and full-constructive crossovers' results for SSC, SAC, NSM and SC are shown in Tables 12 and 13, respectively. It can be seen from Table 12 that while NSM is only slightly more semi-constructive than SC, both SSC and SAC were more semi-constructive than SC and NSM. This is a consequence of the greater semantic diversity of SAC and SSC relative to SC and NSM. Usually, the semi-constructive crossover rate of SAC is from 5 to $7 \%$ higher than $\mathrm{SC}$, and of SSC from 12 to $18 \%$ higher. These increases are particularly important because the semi-constructive rate for SC was fairly small (about 20\%).

Table 13 shows how difficult it is for GP standard crossover to generate improved solutions. The percentage of fully constructive crossovers for SC and NSM were roughly the same, at only $2 \%$ for one-variable functions and $3 \%$ for bivariate functions. By adding semantics to control the crossover operator, far more full-constructive behaviour is obtained. SAC often scored 1.5 times higher than SC and NSM in frequency of full-constructive events, and SSC around 2 to 3 times higher. SSC generated more full-constructive events than SAC (up to 1.5 times) on both univariate and bivariate functions.

\section{Conclusions}

In this paper, we have proposed a new method for measuring semantics of realvalued symbolic regression problems, which we called Sampling Semantics (SS). 
Table 13 The percentage of full-constructive crossovers of SC, NSM, SAC, and SSC (i.e. where both children are better than the corresponding parents)

\begin{tabular}{lllllllllll}
\hline Ms & F1 & F2 & F3 & F4 & F5 & F6 & F7 & F8 & F9 & F10 \\
\hline SC & 2.06 & 2.27 & 2.23 & 2.24 & 1.96 & 2.32 & 2.36 & 1.88 & 3.46 & 3.74 \\
NSM & 2.26 & 2.46 & 2.37 & 2.66 & 1.97 & 2.56 & 2.59 & 2.15 & 3.90 & 3.78 \\
SAC1 & 2.82 & 3.04 & 3.05 & 3.02 & 2.46 & 3.11 & 3.25 & 2.65 & 4.51 & 4.67 \\
SAC2 & 2.90 & 3.04 & 3.29 & 3.25 & 2.67 & 3.24 & 3.49 & 2.89 & 4.95 & 4.87 \\
SAC3 & 3.05 & 3.10 & 3.29 & 3.36 & 2.65 & 3.35 & 3.32 & 2.81 & 4.94 & 4.91 \\
SAC4 & 3.03 & 3.17 & 3.25 & 3.28 & 2.57 & 3.39 & 3.29 & 2.82 & 4.96 & 4.94 \\
SAC5 & 3.01 & 3.18 & 3.20 & 3.31 & 2.52 & 3.43 & 3.28 & 2.82 & 4.92 & 4.92 \\
SSC4 & 3.60 & 3.80 & 3.78 & 3.92 & 3.20 & 3.94 & 3.63 & 3.07 & 4.68 & 5.18 \\
SSC8 & 4.25 & 4.58 & 4.54 & 4.65 & 3.96 & 5.11 & 4.53 & 3.89 & 5.17 & 6.04 \\
SSC12 & 4.70 & 5.21 & 5.07 & 5.02 & 4.51 & 5.38 & 4.88 & 4.43 & 5.45 & 6.35 \\
SSC16 & 4.76 & 5.55 & 5.26 & 5.49 & 4.65 & 5.73 & 5.12 & 4.85 & 6.00 & 6.34 \\
SSC20 & 4.73 & 5.34 & 5.39 & 5.47 & 4.86 & 5.49 & 5.01 & 4.73 & 5.86 & 6.31 \\
\hline
\end{tabular}

Using it, we can define the semantic distance between two subtrees; we then proposed two semantic relations (Semantic Equivalence and Semantic Similarity) for determining the semantic acceptability of exchanging two subtrees. These semantic relations are used to guide crossover, resulting in two new semantically based crossover operators for GP: Semantics Aware Crossover (SAC) and Semantic Similarity-based Crossover (SSC). The new operators were tested on a class of realvalued symbolic regression problems and compared with some similar schemas including No Same Mate (NSM), Soft Brood Selection (SBS) and Context Aware Crossover (CAC), as well as standard GP crossover (SC). On a wide range of problems, only SSC and SBS were consistently better than SC, and of them SSC is the most effective crossover operator.

We also investigated the effect of various parameters on SSC to determine ranges of suitable values. Some characteristics of SSC were analysed, showing that both SAC and SSC improve the resulting semantic diversity. We showed that SSC achieves higher locality than either SAC, NSM or SC. We argue that this is the main reason for its better constructive effect compared to SAC, NSM and SC. This results in a substantial, and statistically significant, improvement in performance of SSC, while SAC and NSM generate almost equivalent performance to SC.

\subsection{Assumptions and limitations}

Although this paper has shown that many benefits are to be gained from incorporating semantics into the design of crossover operators for GP, there are some limitations. First, the paper focuses on the domain of real-valued problems, leaving other domains an open question. ${ }^{16}$ Second, the semantic sensitivities were experimentally determined and might not be the best choices either for these

\footnotetext{
${ }^{16}$ In fact one simple way to use our method is to transform boolean function learning problems to realvalued ones as in [54].
} 
problems, and/or for others. Adaptive mechanisms to determine these values are currently under investigation.

We hypothesised that fixed semantics might occur in real-valued trees as in Boolean trees, but further studies need to be conducted to understand whether fixed semantics really occurs, and if so, what form it takes in real-valued problem domains. More importantly, by advocating for semantic locality in GP crossover operators, we have assumed that there is a smooth correlation between semantics of subtrees to the semantics of the whole individual tree and to the overall fitness of the individual. This might limit the type of problems that our SSC would excel. For some problems that do not satisfy this assumption, non-locality of semantic (fitness) caused by crossovers might be needed. However, we argue that such problems would not be beneficial to GP or any type of evolutionary algorithms as the change in a phenotype would be unpredictable with any small change in the corresponding genotype. Such search fitness landscape would be too rough for any progression search techniques, such as GP, to be better than random search.

\section{Future work}

In the near future, we plan to extend this work in a number of ways. First, we will apply SSC to some more difficult symbolic regression problems (problems that have more complex-structured solutions). For these problems, we predict that making small changes in semantics will be both more difficult, but also more important. Second, SSC could be used to enhance some previously proposed crossover operators, which are based purely on the structure of trees-such as crossover with bias on the depth of nodes [24], one point crossover and uniform crossover [48, 39]. Another potential research direction is to apply SSC to other problem domains, such as the Boolean problems that have been previously investigated in [43]. In this case, it may be even more difficult to generate children that differ semantically from their parents, so that the benefits may be greater. Last but not least, we plan to investigate suitable ranges for the lower and upper bound semantic sensitivity values for various classes of problems. In this paper, these values were manually and experimentally specified; however, it may be possible to allow these values to self-adapt during the evolutionary process [14].

Acknowledgements This paper was funded under a Postgraduate Scholarship from the Irish Research Council for Science Engineering and Technology (IRCSET). The authors would like to thank the members of NCRA (Natural Computing Research \& Applications Group) at University College Dublin. The second author was partly funded by The Vietnam National Foundation for Science and Technology Development (NAFOSTED) under grant number 102.01.14.09 for doing this work.

\section{References}

1. C. Alan, Meaning and language: an introduction to semantics and pragmatics. (Oxford Textbooks in Linguistics, Cambridge, 2004)

2. L. Altenberg, in Advances in Genetic Programming, ed. by K. E. Kinnear, Jr., The evolution of evolvability in genetic programming, chap. 3 (MIT Press, Cambridge, 1994), pp. 47-74 
3. C. Baier, J.P. Katoen, Principles of Model Checking (MIT Press, Cambridge, 2008).

4. L. Beadle, C. Johnson, Semantically driven crossover in genetic programming, in Proceedings of the IEEE World Congress on Computational Intelligence (IEEE Press, New York, 2008), pp. 111-116

5. L. Beadle, C.G. Johnson, Semantic analysis of program initialisation in genetic programming. Genet. Program. Evol. Mach. 10(3), 307-337 (2009)

6. L. Beadle, C. G. Johnson, Semantically driven mutation in genetic programming. in 2009 IEEE Congress on Evolutionary Computation, ed. by A. Tyrrell (IEEE Computational Intelligence Society, IEEE Press, Trondheim, Norway, 18-21 May 2009), pp. 1336-1342

7. R.E. Bryant, Graph-based algorithms for Boolean function manipulation. IEEE Trans. Comp. C-35, 677-691 (1986)

8. E.K. Burke, S. Gustafson, G. Kendall, Diversity in genetic programming: an analysis of measures and correlation with fitness. IEEE Trans. Evol. Comput. 8(1), 47-62 (2004)

9. R. Cleary, M. O'Neill, Solving knapsack problems with attribute grammars, in Proceedings of the Grammatical Evolution Workshop, 2004

10. R. Cleary, M. O'Neill, in Proceedings of the Evolutionary Computation in Combinatorial Optimization. An attribute grammar decoder for the 01 multi-constrained knapsack problem (Springer, Berlin, 2005), pp. 34-45

11. A.M. Collins, M.R. Quillian, Retrieval time from semantic memory. J. Verbal Learn.Verbal Behav. 8, 240-247 (1969)

12. J.M. Daida, D.S. Ampy, M. Ratanasavetavadhana, H. Li, O. Chaudhri, in Proceedings of the Genetic and Evolutionary Computation Conference, (GECCO'1999). Challenges with verification, repeatability, and meaningful comparison in genetic programming: Gibson's magic (Morgan Kaufmann, 1999), pp. 1851-1858

13. M. de la Cruz Echeand'a, A.O. de la Puente, M. Alfonseca, in Proceedings of the IWINAC 2005. Attribute grammar evolution (Springer, Berlin, 2005), pp. 182-191

14. K. Deb, H.G. Beyer, in Proceedings of the Genetic and Evolutionary Computation Conference. Selfadaptation in real-parameter genetic algorithms with simulated binary crossover (Morgan Kaufmann, July 1999), pp. 172-179

15. E. Galvan-Lopez, M. O'Neill, in CIG. On the effects of locality in a permutation problem: the sudoku problem (IEEE, 2009)

16. E. Galvan-Lopez, M. O'Neill, in MICAI, Lecture Notes in Computer Science. Towards understanding the effects of locality in genetic programming (Springer, Berlin, 2009)

17. J. Gottlieb, G. Raidl, in Proceedings of the Genetic and Evolutionary Computation Conference. The effects of locality on the dynamics of decoder-based evolutionary search (ACM, 2000), p. 283-290

18. S. Gustafson, E.K. Burke, N. Krasnogor, in Proceedings of the 2005 IEEE Congress on Evolutionary Computation. On improving genetic programming for symbolic regression, vol. 1. (IEEE Press, Edinburgh, 2005), pp. 912-919

19. S. Hengpraprohm, P. Chongstitvatana, in Proceedings of ISCIT International Symposium on Communications and Information Technologies. Selective crossover in genetic programming, Nov 2001, pp. $14-16$

20. N.X. Hoai, R. McKay, D. Essam, in Proceedings of the 2002 Congress on Evolutionary Computation (CEC2002). Solving the symbolic regression problem with tree-adjunct grammar guided genetic programming: the comparative results (IEEE Press, 2002), pp. 1326-1331

21. N.X. Hoai, R.I. McKay, D. Essam, Representation and structural difficulty in genetic programming. IEEE Trans. Evol. Comput. 10(2), 157-166 (2006)

22. N.X. Hoai, R.I.B. McKay, D. Essam, H. Abbass, in Genetic Programming 7th European Conference, EuroGP 2004, Proceedings, vol. 3003 of LNCS, ed. by M. Keijzer, U.-M. O'Reilly, S.M. Lucas, E. Costa, T. Soule, Toward an alternative comparison between different genetic programming systems (Springer, Berlin, 2004), pp. 67-77

23. T.-H. Hoang, D. Essam, R.I.B. McKay, X.H. Nguyen, in Proceedings of the 2007 International Symposium on Intelligent Computation and Applications (ISICA). Building on success in genetic programming:adaptive variation \& developmental evaluation (China University of Geosciences Press, Wuhan, China, Sep 2007)

24. T. Ito, H. Iba, S. Sato, in Proceedings of the 1998 IEEE World Congress on Computational Intelligence. Depth-dependent crossover for genetic programming (IEEE Press, May 1998), pp. 775-780

25. T. Ito, H. Iba, S. Sato, in Advances in Genetic Programming. A self-tuning mechanism for depthdependent crossover (IEEE Press, June 1999), pp. 377-399 
26. C. Johnson, in Proceedings of the 4th European Conference on Genetic Programming (EuroGP2002). Deriving genetic programming fitness properties by static analysis (Springer, Berlin, 2002), pp. $299-308$

27. C. Johnson, in Recent Advances in Soft Computing. Genetic programming with guaranteed constraints (The Nottingham Trent University, UK, 2002), pp. 134-140

28. C. Johnson, in Proceedings of the UK Workshop on Computational Intelligence. What can automatic programming learn from theoretical computer science (University of Birmingham, Birmingham, 2002)

29. C. Johnson, in Proceedings of the 10th European Conference on Genetic Programming (EuroGP2002). Genetic programming with fitness based on model checking (Springer, Berlin, 2007), pp. 114-124

30. C. Johnson, in Proceedings of the 12th European Conference on Genetic Programming (EuroGP2009). Genetic programming crossover: Does it cross over? (Springer, Berlin, 2009), pp. 97-108

31. G. Katz, D. Peled, Genetic programming and model checking: Synthesizing new mutual exclusion algorithms. Automated technology for verification and analysis. Lect. Notes Comput. Sci. 5311, 33-47 (2008)

32. G. Katz, D. Peled, Model checking-based genetic programming with an application to mutual exclusion. Tools Algorithm. Constr. Anal. Syst. 4963, 141-156 (2008)

33. M. Keijzer, in Proceedings of EuroGP'2003. Improving symbolic regression with interval arithmetic and linear scaling, Springer, Berlin, April 2003), pp. 70-82

34. D. Knuth, Semantics of context-free languages. Math. Syst. Theory. 295 (1968)

35. J. Koza, Genetic Programming: On the Programming of Computers by Natural Selection (MIT Press, Cambridge, 1992)

36. J.R. Koza, Genetic Programming: On the Programming of Computers by Means of Natural Selection (The MIT Press, Cambridge, 1992)

37. K. Krawiec, P. Lichocki, in Genetic and Evolutionary Computation Conference, GECCO 2009, Proceedings, Montreal, Québec, Canada, July 8-12, 2009, ed. by F. Rothlauf. Approximating geometric crossover in semantic space (ACM, New York, 2009), pp. 987-994

38. K. Krawiec, B. Wieloch, in GECCO '09: Proceedings of the 11th Annual conference on Genetic and evolutionary computation. Functional modularity for genetic programming (ACM, Montreal, July 2009), pp. 995-1002

39. W.B. Langdon, in Proceedings of the Genetic and Evolutionary Computation Conference. Size fair and homologous tree genetic programming crossovers (Morgan Kaufmann, July 1999), pp. 1092-1097

40. W. B. Langdon, R. Poli, Foundations of Genetic Programming (Springer, Berlin, 2002)

41. H. Majeed, C. Ryan, in Proceedings of the 9th European Conference on Genetic Programming. A less destructive, context-aware crossover operator for gp, Lecture Notes in Computer Science (Springer, Berlin, April 2006), pp. 36-48

42. H. Majeed, C. Ryan, in Proceedings of the 9th Annual Conference on Genetic and Evolutionary Computation (GECCO). On the constructiveness of context-aware crossover (ACM Press, New York, July 2007), pp. 1659-1666

43. N. McPhee, B. Ohs, T. Hutchison, in Proceedings of 11th European Conference on Genetic Programming. Semantic building blocks in genetic programming (Springer, Berlin, 2008), pp. 134-145

44. N. Mori, B. McKay, N.X. Hoai, D. Essam, S. Takeuchi, A new method for simplifying algebraic expressions in genetic programming called equivalent decision simplification. J. Adv. Comput. Intell. Intell. Inform. 13(3), 237-244 (2009)

45. F. Nielson, H.R. Nielson, C. Hankin, Principles of Program Analysis. (Springer, Berlin, 2005)

46. H.R. Nielson, F. Nielson, Semantics with Applications: An Appetizer (Springer, London, 2007)

47. U.M. O'Reilly, F. Oppacher, Program search with a hierarchical variable length representation: genetic programming, simulated annealing and hill climbing. Lect. Notes Comput. Sci. 866(1), 397-406 (1994)

48. R. Poli, W.B. Langdon, in Proceedings of Soft Computing in Engineering Design and Manufacturing Conference. Genetic programming with one-point crossover (Springer, Berlin, June 1997), pp. 180-189

49. R. Poli, W.B. Langdon, N.F. McPhee, A Field Guide to Genetic Programming. Published via http://lulu.com and freely available at http://www.gp-field-guide.org.uk, 2008. (With contributions by J. R. Koza).

50. B.J. Ross, Logic-based genetic programming with definite clause translation grammars. New Gen. Comput. 19(4), 313-337 (2001)

51. F. Rothlauf, Representations for Genetic and Evolutionary Algorithms, 2nd edn. (Springer, Berlin, 2006) 
52. F. Rothlauf, D. Goldberg, Redundant representations in evolutionary algorithms. Evol. Comput. 11(4), 381-415 (2003)

53. F. Rothlauf, M. Oetzel, in Proceedings of the 9th European Conference on Genetic Programming. On the locality of grammatical evolution, lecture notes in computer science (Springer, Berlin, April 2006), pp. 320-330

54. R.P. Salustowicz, J. Schmidhuber, Probabilistic incremental program evolution. Evol. Comput. 5(2), 123-141 (1997)

55. W.A. Tackett, Selection, and the Genetic Construction of Computer Programs. PhD thesis, University of Southern California, USA, 1994

56. W.A. Tackett, A. Carmi, in Proceedings of the 1994 IEEE World Congress on Computational Intelligence. The unique implications of brood selection for genetic programming (IEEE Press, Orlando, Florida, USA, 27-29 June 1994)

57. N.Q. Uy, N.X. Hoai, M. O’Neill, in Proceedings of EuroGP09. Semantic aware crossover for genetic programming: the case for real-valued function regression (Springer, Berlin, April 2009), pp. 292-302.

58. M.L. Wong, K.S. Leung, in Proceedings of the 7th IEEE International Conference on Tools with Artificial Intelligence. An induction system that learns programs in different programming languages using genetic programming and logic grammars (1995)

59. M.L. Wong, K.S. Leung, in Proceedings of the Fourth Congress of the Italian Association for Artificial Intelligence. Learning programs in different paradigms using genetic programming (Springer, Berlin, 1995)

60. P. Wong, M. Zhang, 2008 IEEE World Congress on Computational Intelligence, ed. by J. Wang. SCHEME: caching subtrees in genetic programming (IEEE Computational Intelligence Society, IEEE Press, Hong Kong, 1-6 June 2008) 